\title{
Treatment of Metastasized Breast Cancer
}

\author{
Hans-Joachim Lück \\ Gynäkologisch-Onkologische Praxis Hannover, Germany
}

Metastatic spread is a fact in breast cancer: nearly $25 \%$ of women with breast cancer develop distant metastasis and at least $60 \%$ of breast cancers are hormone receptor-positive. These patients are commonly treated with endocrine therapy in addition to adjuvant treatment. In patients who do not respond to endocrine therapy, or in patients with hormone receptor-negative tumors, chemotherapy is the first choice of treatment.

Over the last 10 years new therapeutic strategies were developed additionally to the common therapeutic options, namely antibodies and tyrosine kinase inhibitors (TKI). The first monoclonal humanized antibody in breast cancer treatment was trastuzumab. This antibody binds to the extracellular domain of HER2, and was able to show an impressive activity in metastatic cancer. Over time, other antibodies with different specificities, such as bevacizumab, T-DM1 or pertuzumab, were developed.

TKI as a treatment option came up in the beginning 1990ies. These drugs were highly specific and less toxic than chemotherapies. However, several phase III studies were not able to show a significant advantage over the common standards of this time. The new generations of TKI are multifunc- tional receptor inhibitors. With these the efficacy seems to be better, while on the other hand the toxicity is more pronounced. For both new options the side effects are different from endocrine or chemotherapy. Cardiac events, skin toxicity, changes in blood pressure, and gastrointestinal problems are relatively common for these treatments.

Still, the 'old-fashioned' chemotherapy is not outdated: the new options mostly developed their best activity in combination with chemotherapy. In cases where we do not have the option to use targeted therapy, chemotherapy is the only way that works. Dependent on the number and intensity of symptoms, according the national and international guidelines mono- or combination therapies are used.

There is long-standing discussion which kind of chemotherapeutic strategy is the best. It looks like over the time of treatment there is no difference in outcome between sequential monotherapy and combination therapies, but the risk for severe side effects is higher with combinations. However, all these results come from studies that did not incorporate the new treatment options.

This issue should give an overview on what is going on in this field, and should give perspectives for the future.

\section{KARGER}

Fax +497614520714

Information@Karger.de

www.karger.com (c) 2009 S. Karger GmbH, Freiburg
Prof. Dr. med. Hans-Joachim Lück

Gynäkologisch-Onkologische Praxis Hannover

Pelikanplatz 23

30177 Hannover, Germany

Tel. +49 511 6555-280, Fax -2898

info@go-praxis-hannover.de 